Universitas, Volumen 1, Número 2, 2008, 31-36

๑ 2008 UNAN-León, Editorial Universitaria

\title{
Efecto de factores físicos y bioquímicos sobre la formación de estructuras infectivas de Magnaporthe grisea agente causal de pyriculiarosis en arroz (Oryza sativa)
}

\author{
Wilber Salazar Antón* \\ Area de Fitopatología. Departamento de Agroecología. Universidad Nacional Autónoma de Nicaragua, León \\ (UNAN-León).
}

\section{RESUMEN}

\begin{abstract}
Este estudio se realizó en el laboratorio de Fitopatología de la Universidad de Kobe, Japón, evaluándose el efecto de dos factores físicos (superficies hidrofóbicas e hidrofílicas) y dos factores químicos (Adenosin Monofosfato cíclico (cAMP) y colagenasa) sobre la capacidad de adhesión y producción de apresorios de Magnaporthe grisea sobre superficies hidrofóbicas e hidrofílicas. En este estudio la concentración de conidias fue ajustada a $1 \times 10^{4}$ conidias/mililitro y se utilizó plástico Gelbond ${ }^{\mathrm{TM}}$ Film. Los resultados indican que sobre superficies hidrofóbicas el $78 \%$ de conidias produjeron apresorios, reduciéndose este porcentaje a cero cuando fueron colocadas sobre una superficie hidrofílica. Sin embargo, la adición de cAMP a las conidias creciendo sobre superficies hidrofílicas les restituyó la capacidad de producir apresorios hasta $69 \%$. Cuando se aplicó colagenasa a la solución de conidias después de 6 horas de incubación su capacidad de adhesión se redujo hasta $61 \%$ en comparación con el tratamiento testigo sin colagenasa. Esta reducción fue más evidente cuando la solución de conidias se incubó por 6 horas junto con la colagenasa, reduciéndose el número de conidias adheridas a $97 \%$ en comparación con el testigo. La colagenasa también redujo la severidad de los síntomas de M. grisea en plántulas de arroz. La formación de estructuras infectivas de M. grisea se ve favorecida por superficies hidrofóbicas y la presencia de cAMP, en cambio, las superficies hidrofílicas y la colagenasa las inhiben.
\end{abstract}

Palabras claves: cAMP; Colagenasa; Apresorio; Hidrofóbica; Hidrofílica.

\section{INTRODUCCIONN}

El cultivo del arroz (Oryza sativa) es una de las principales fuentes de alimentos para la mayoría de países en desarrollo. Este cultivo aporta un $20 \%$ de calorías y $13 \%$ de las proteínas consumidas en el mundo ${ }^{[14]}$. La cantidad de productos alimenticios derivados de arroz consumidos por las poblaciones de países en desarrollo oscila entre 100-200 kg per capita por año ${ }^{[7]}$.

Adicionalmente a este alto consumo de arroz, se estima que la población mundial crecerá hasta 8.2 billones de personas para el año 2030. Esto significa que la demanda mundial de arroz se incrementará en aproximadamente 533 millones de toneladas de arroz ${ }^{[7]}$.

La producción de arroz tiene muchos factores que limitan su rendimiento. Entre estos factores sobresalen insectos plagas, malezas y enfermedades. Sin embargo, es la enfermedad conocida como pyriculariosis causado por Magnaporthe oryzae Couch (anamorph Pyricularia oryzae Cav.), la que ha sido reportada como la más destructiva en el cultivo del arroz, especialmente en áreas donde se utiliza altos niveles de nitrógeno en la fertilización del cultivo, causando altas pérdidas en los rendimientos ${ }^{[1]}$.

Durante el año 2003, en la India, la pyriculiarosis fue responsable de pérdidas de 266,000 toneladas de arroz, lo cual representa cerca del $8 \%$ de la producción arrocera del país.

\footnotetext{
*Autor para correspondencia: wsalazar_a@yahoo.com
}

En Japón, la enfermedad afecta anualmente aproximadamente 865,00 hectáreas de arroz cada año. En Filipinas, los productores de arroz sufren mas de $50 \%$ de pérdidas cada año causados por esta misma enfermedad ${ }^{[9]}$. En Nicaragua, existe muy poca información en la literatura sobre las pérdidas causadas por este hongo en los campos arroceros, sin embargo, los productores de arroz han reportado reducción en sus rendimientos a causa de esta enfermedad.

El hongo $M$. grisea, al igual que muchos otros hongos fitopatógenos, necesita para causar infección la formación de apresorios, los cuales son estructuras infectivas útiles para adherirse fuertemente a la superficie de su hospedero y luego penetrarlo y colonizarlo. La formación de esta estructura es inducida por estímulos ambientales que incluyen factores químicos como la presencia de cAMP y físicos como las características hidrofóbicas presentes en las superficies de la planta $y$ el hongo ${ }^{[8,13]}$.

A pesar de la alta correlación existente entre la formación de estructuras infectivas y la patogenicidad de Magnaporthe grisea, existen muy pocos estudios que, identifiquen y evalúen los factores que se ven involucrados en los procesos pre-infección. Es evidente que un mejor entendimiento de los fenómenos que preceden la infección de las plantas por patógenos nos permitirá desarrollar un mejor sistema de manejo de esta enfermedad que sea más durable y ambientalmente amigable. 
Este estudio pretende mejorar el entendimiento actual sobre los mecanismos utilizados por este patógeno para adherirse, causar infección y penetrar en sus plantas hospederas.

\section{MATERIALES Y MÉTODOS}

El presente estudio se llevó a cabo en el laboratorio de Fitopatología de la Universidad de Kobe en Japón, durante el periodo de mayo a septiembre de 2007.

\subsection{Preparación de la suspensión de conidias}

El hongo utilizado en este estudio fue Magnaporthe grisea, el cual es patogénico para el cultivo del arroz. Este hongo fue mantenido en un medio modificado el cual consistió en $20 \mathrm{~g}$ de avena (Quaker), $10 \mathrm{~g}$ de agar y $500 \mathrm{~mL}$ de agua destilada, con el fin de obtener un crecimiento denso del hongo. Posteriormente, el hongo fue incubado a $24^{\circ} \mathrm{C}$ por nueve días, después de este periodo de incubación, los platos Petri, conteniendo el hongo, fueron lavados y removidos con una espátula.

Una vez que, las estructuras superficiales del hongo fueron lavadas, los platos Petri fueron expuestos a una luz cercana a la luz ultravioleta $(360 \mathrm{~nm})$ a $24^{\circ} \mathrm{C}$ por tres días. Después de incubar el hongo y exponerlo a radiación, se agregó agua estéril al medio de cultivo antes descrito y se removieron las esporas que se produjeron durante los tres días de incubación. Luego la suspensión de conidias fue filtrada con papel toalla y centrifugada a 3,000 rpm por 10 minutos. La concentración de conidias utilizadas fue de $1 \times 10^{4}$ conidias $/ \mathrm{mL}$.

\subsection{Efecto de cAMP, superficies hidrofílicas e hidrofóbicas sobre la capacidad de adhesión de conidias de M. grisea}

Se utilizaron trozos de plástico (Gelbond ${ }^{\mathrm{TM}}$ film), el cual posee una superficie hidrofóbica y una superficie hidrofílica, El plástico fue cortado en 15 trozos de aproximadamente $3 \mathrm{~cm}^{2}$, utilizándose las superficie hidrofílica en 10 trozos y la superficie hidrofóbico en los restantes cinco trozos. Esto se realizó con el fin de determinar la capacidad de adhesión de las conidias a estas dos superficies. Se utilizó cAMP obtenido en Elastin Products Co., Missouri, USA.

Se evaluaron tres tratamientos los cuales fueron, 1) superficie hidrofílica; 2) superficie hidrofóbica y 3) superficie hidrofílica + cAMP. Los tratamientos 1 y 2 recibieron cuatro gotas separadas de $10 \mu \mathrm{L}$ de la solución conteniendo conidias. El tratamiento 3 recibió cuatro gotas separadas de $10 \mu \mathrm{L}$ de la solución de conidias, a las cuales se les adicionó $10 \mu \mathrm{L}$ de cAMP. Los tratamientos evaluados en este experimento fueron repetidos cuatro veces. Las mediciones de los tratamientos evaluados se realizaron después de incubarse por 1, 3 y 6 horas en una cámara húmeda a $26^{\circ} \mathrm{C}$. Los porcentajes de conidias germinadas, y formando apresorios fue determinada a través de observación directa en el microscopio, de 100 conidias por cada repetición, 500 conidias por tratamiento.

2.3. Efecto inhibitorio de la enzima colagenasa sobre la adhesión de conidias de Magnaporthe grisea a una superficie hidrofóbica y patogenicidad sobre plantas de arroz.

En este estudio, se empleó trozos de plástico Gelbond $^{\mathrm{TM}}$ film, el cual fue cortado en 18 trozos de aproximadamente $3 \mathrm{~cm}^{2}$. Se colocaron tres gotas de $10 \mu \mathrm{L}$ de la suspensión de conidias separadamente en cada trozo de plástico utilizando su lado hidrofóbico.

El primer tratamiento consistió en seis trozos de plástico conteniendo la solución de conidias, los cuales fueron inoculados con $10 \mu \mathrm{L}$ colagenasa (CL103, Elastin Products Co., Missouri, USA). El segundo tratamiento, consistió en 6 trozos de plástico conteniendo la solución de conidias a los cuales después de incubarse se aplicó la enzima colagenasa. Finalmente, como control se utilizaron seis trozos de plástico conteniendo únicamente la solución de conidias. Todos los trozos de plástico Gelbond $^{\mathrm{TM}}$ film, conteniendo la solución de conidias, fueron colocados en papel toalla húmeda dentro de un plato Petri por seis horas a $26^{\circ} \mathrm{C}$.

Posterior al periodo de incubación, cada trozo de plástico Gelbond ${ }^{\mathrm{TM}}$ film, conteniendo la solución de conidias, fue lavado en agua destilada sumergiéndolos 100 veces verticalmente. Los porcentajes de conidias adheridas a la superficie artificial utilizadas, fue determinada a través de observación directa en el microscopio de 100 conidias por cada repetición, 600 conidias por tratamiento.

\subsection{Efecto de la enzima colagenasa en la patogénesis del hongo Magnaporthe grisea en plántulas de arroz}

Se compararon dos tratamientos; el primero fue la aplicación de solución de conidias a plántulas de arroz. El segundo fue la aplicación de solución de conidias + colagenasa (CL103, Elastin Products Co., Missouri, USA) a plántulas de arroz y finalmente un control con agua destilada. Cinco plántulas fueron usadas en cada una de las cinco repeticiones del estudio, las cuales fueron numeradas del uno al cinco. 
Las primeras dos plántulas fueron inoculadas con la solución de conidias, la tercer plántula fue inoculada con agua estéril y la cuarta y quinta plántula fueron inoculadas con solución de conidias + colagenasa. La solución de conidias fue inoculada sobre las hojas de plántulas de arroz, dispensando con una micropipeta 3 gotas de $10 \mu \mathrm{L}$ de solución conteniendo conidias en la parte apical de la hoja de las plántulas.

Cinco días después de la inoculación del hongo en la planta, fue evaluada la severidad de la infección utilizando una escala de $0-5$ : 0 , no síntoma visible; 1 , pequeños puntos café; 2 , lesiones pequeñas café; 3 , lesiones intermedias; 4 lesiones grandes; 5 lámina foliar completamente marchita.

\section{Hospedero}

Las plántulas de arroz utilizadas en este estudio (Oryza sativa var. Japonica) son susceptibles a $M$. grisea. Las semillas de arroz fueron pre germinadas antes de sembrarlas con el fin de asegurar una germinación uniforme. Este proceso, se llevó a cabo incubando semillas de arroz en papel filtro húmedo colocado dentro de un plato Petri por 24 horas a $26^{\circ} \mathrm{C}$.

Las semillas germinadas se sembraron en vermiculita, en recipientes de $5.5 \times 15 \times 10 \mathrm{~cm}$. Luego las plántulas, fueron mantenidas a $26^{\circ} \mathrm{C}$ por 10 días, en una cámara de crecimiento expuesta a 12 horas de fotoperíodo hasta que desarrollaron su primera hoja.

\section{RESULTADOS Y DISCUSIÓN}

\subsection{Efecto de CAMP, superficies hidrofílicas e hidrofóbicas sobre la capacidad de adhesión de conidias de $M$. grisea}

La formación de estructuras infectivas producidas por el hongo fue evidentemente afectada por la superficie en la cual la conidia estaba creciendo. Después de 6 horas de incubación en agua destilada, las conidias que fueron depositadas en superficies hidrofílicas no produjeron apresorios. Sin embargo, el $78 \%$ de las conidias colocadas en una superficie hidrofóbica fueron capaces de producir apresorios después de 6 horas de incubación (Tabla 1).

Los resultados obtenidos sugieren que el tipo de superficie presente al momento en que el proceso infeccioso inicia, juega un papel muy importante en la formación de estructuras infectivas tales como apresorios. Otros autores han logrado comprobar que superficies hidrofóbicas, comunes en las hojas de las plantas, inducen la formación de apresorios, no así las superficies hidrofílicas las cuales son incapaces de formar dichas estructuras en hongos fitopatógenos, tales como Colletotrichum graminicola ${ }^{[5]}$.

En el caso particular del hongo Magnaporthe grisea, los resultados en este estudio coinciden con los obtenidos por Lee y Dean $^{[9]}$ quienes afirman que las conidias germinadas de $M$. grisea, forman tubos germinativos y apresorios eficientemente en superficies hidrofóbicas pero no en estructuras hidrofílicas.

El porcentaje de conidias formando apresorios, obtenido en la superficie hidrofílica variaron significativamente cuando la solución conteniendo las conidias fue tratada con AMP cíclico. En este caso, el porcentaje de conidias formando apresorios se incrementó de 0-69\%.

Los resultados obtenidos, coinciden con lo reportado por otros autores, quienes afirman que cAMP induce la formación de apresorios en superficies hidrofílicas en las que normalmente la conidia germinada no produce apresorios. Esto sugiere, el involucramiento de cAMP en la formación de apresorios independientemente de las características hidrofílicas o hidrofóbicas de las superficies $^{[12]}$.(Figura No. 1)

Tabla 1. Efecto de superficies hidrofilicas, hidrofóbicas y cAMP sobre la germinación y formación de apresorios en conidias de Magnaporthe grisea.

\begin{tabular}{|c|c|c|c|}
\hline Superficie & $\begin{array}{c}\text { Tiempo de } \\
\text { incubación }\end{array}$ & $\begin{array}{c}\text { Germinación } \\
\text { de conidas } \\
(\% \pm \mathrm{DE})^{*}\end{array}$ & $\begin{array}{c}\text { Formación } \\
\text { de apresorios } \\
(\% \pm \mathrm{DE})^{*}\end{array}$ \\
\hline \multirow{4}{*}{ Hodrofilica } & 0 horas & $0.0 \pm 0.0$ & $0.0 \pm 0.0$ \\
\cline { 2 - 4 } & 1 horas & $75 \pm 3.93$ & $0.0 \pm 0.0$ \\
\cline { 2 - 4 } & 3 horas & $81 \pm 4.35$ & $0.0 \pm 0.0$ \\
\cline { 2 - 4 } & 6 horas & $85 \pm 4.77$ & $0.0 \pm 0.0$ \\
\hline \multirow{4}{*}{ Hidrofóbica } & 0 horas & $0.0 \pm 0.0$ & $0.0 \pm 0.0$ \\
\cline { 2 - 4 } & 1 horas & $79 \pm 1.58$ & $0.0 \pm 0.0$ \\
\cline { 2 - 4 } & 3 horas & $84 \pm 3.39$ & $49.0 \pm 3.39$ \\
\cline { 2 - 4 } Hidrofilica & 6 horas & $88 \pm 2.44$ & $78.0 \pm 3.31$ \\
\hline +cAMP & 0 horas & $0.0 \pm 0.0$ & $0.0 \pm 0.0$ \\
\cline { 2 - 4 } & 1 horas & $84 \pm 2.73$ & $0.0 \pm 0.0$ \\
\cline { 2 - 4 } & 3 horas & $86 \pm 4.06$ & $41.0 \pm 3.39$ \\
\cline { 2 - 4 } & 6 horas & $92 \pm 3.80$ & $69.0 \pm 4.0$ \\
\hline
\end{tabular}

*\%: Porcentaje

${ }^{*} D E$ : Desviación estándar 


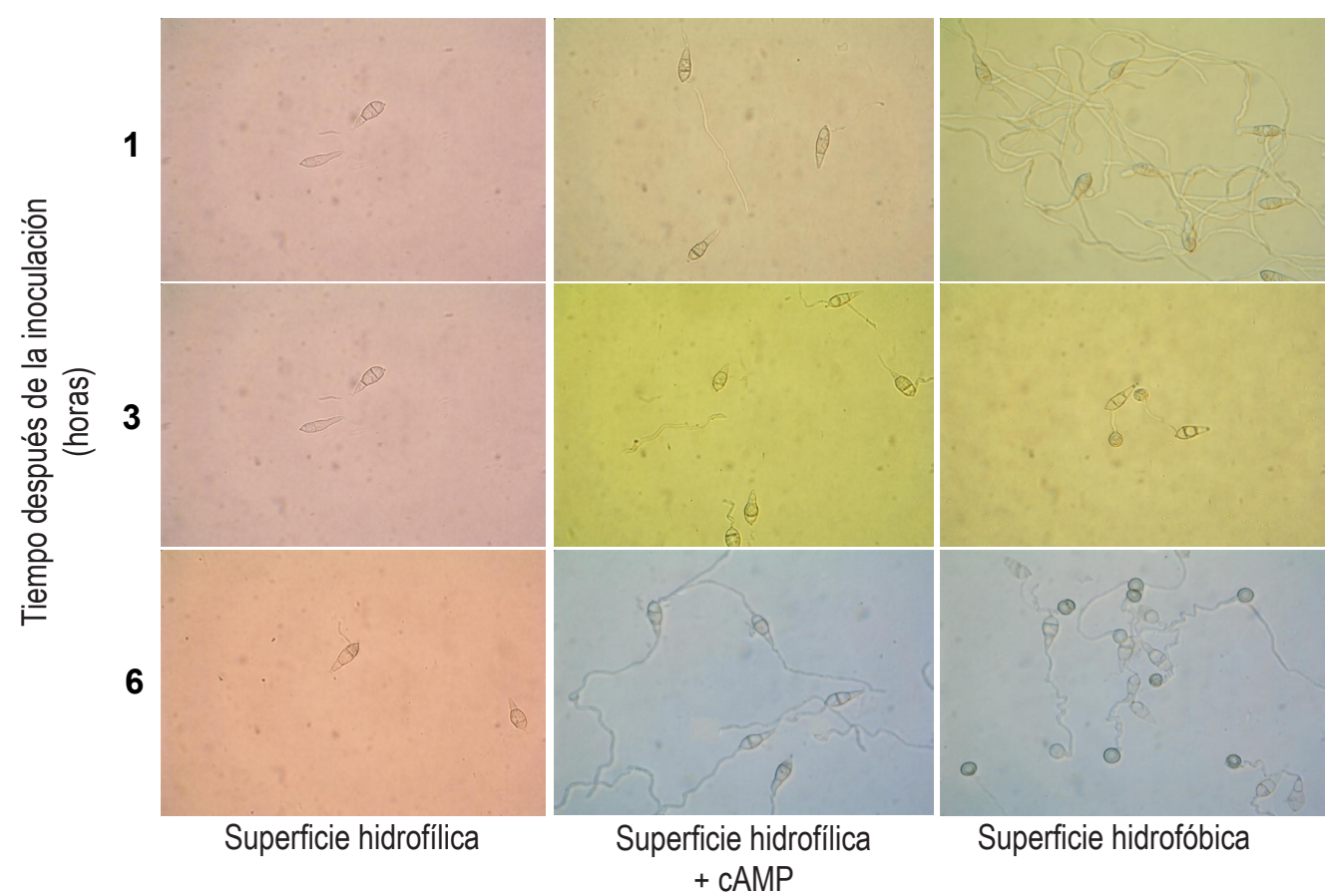

Figura 1. Efecto de superficies hidrofilicas, hidrofilicas + CAMP e hidrofóbicas sobre la formación de apresorios de conidias de M. grisea

3.2. Efecto de la enzima colagenasa sobre la formación de estructuras infectivas y patogénesis de Magnaporthe grisea sobre una superficie hidrofóbica y sobre plantas de arroz.

Los resultados obtenidos en este estudio, indican que la enzima colagenasa, es un factor que influye en la adhesión de las conidias de $M$. grisea. Esto se puede deber, al hecho de que, la adhesión de las conidias a superficies depende de la formación de apresorios los cuales son afectados por la enzima colagenasa. En este estudio, el testigo presentó el más alto porcentaje de conidias adheridas con un promedio de 560. (Figura 2)
En este caso, no se aplicó colagenase a la solución de conidias, de manera que esta cifra indica el porcentaje de conidias que se podrían adherir en condiciones óptimas.

Cuando se aplicó colagenasa a la solución de conidias antes de incubarse, la capacidad de las conidias de adherirse fue reducida. Esto se debe a la acción de la colagenasa la cual degrada las glicoproteinas presentes en las estructuras de los hongos, lo que hace que las conidias del hongo no puedan adherirse. En este tratamiento el número de conidias adheridas fue de 20 que representan un $3 \%$ comparada con el control.

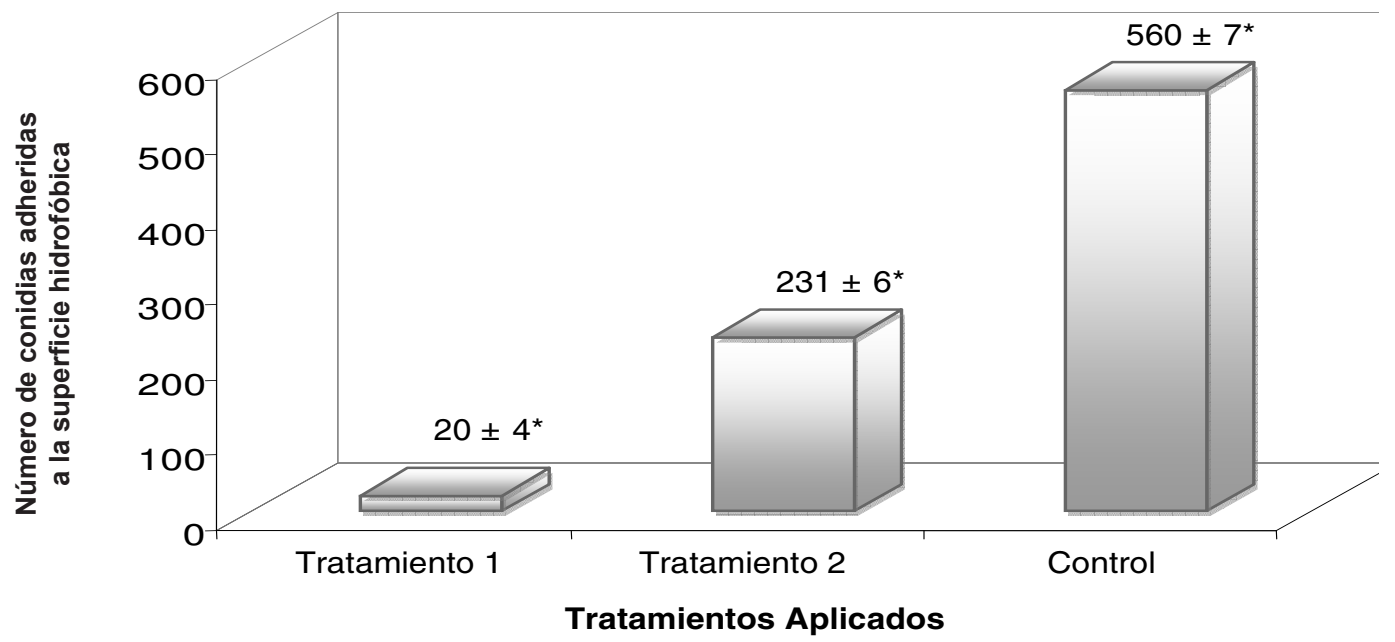

Figura 2. Efecto de la enzima colagenasa sobre la capacidad de adhesión de conidias de Magnaporthe grisea a una superficie artificial hidrofóbica.

* Promedio y Desviacion estandar

Tratamiento 1. Solución de conidias + colagenasa incubada por 6 horas, Tratamiento 2. Solución de conidias incubadas por 6 horas y luego adición de colagenasa, Control. Solución de conidias incubadas por 6 horas en agua destilada. 
El tratamiento número 2, donde la enzima colagenasa fue aplicada a la suspensión de conidias después de ser incubadas por 6 horas, el porcentaje de conidias adheridas fue mas alta que el tratamiento 1 pero más bajo que el control sin colagenasa. Este tratamiento presentó un promedio de 231 conidias adheridas lo cual representa el $41 \%$ del tratamiento control. (Figura 3 )

La razón por la que el tratamiento 2 exhibió un número mayor de conidia adheridas que en el tratamiento 1, fue porque la enzima fue aplicada después que las conidias tenían 6 horas de incubación. Después de seis horas de incubación estructuras infectivas esenciales para que la conidias se adhieran ya están formadas por lo que la acción de la enzima colagenasa tuvo menor incidencia en la capacidad de las conidias para adherirse.

Sin embargo, de acuerdo al presente estudio más de la mitad de las conidias fueron removidas cuando fueron lavadas.

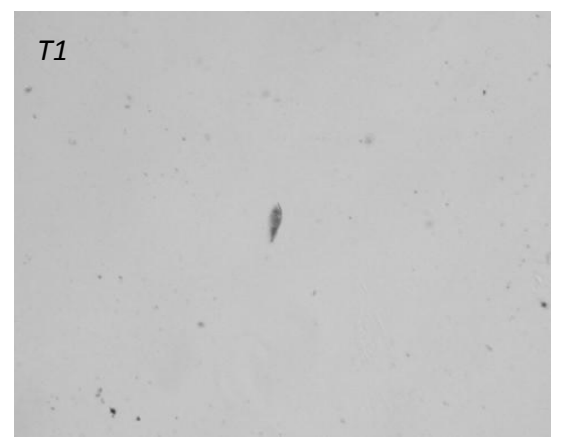

$3 \%$ de conidias adheridas

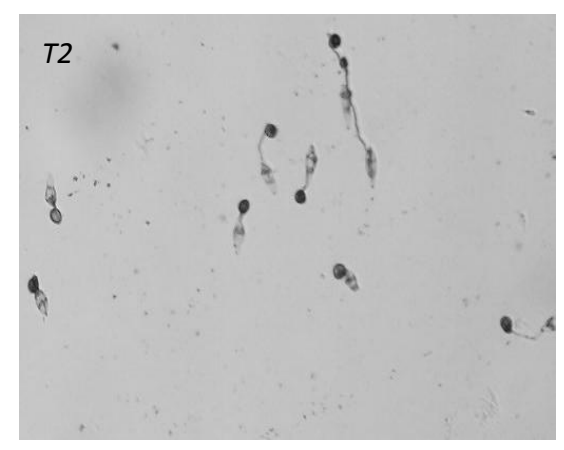

$41 \%$ de conidias adheridas

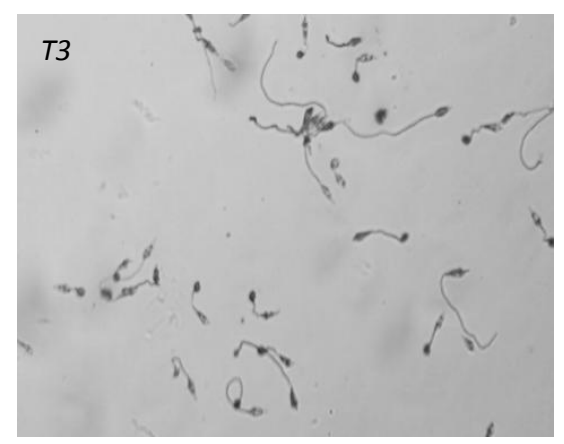

$100 \%$ de conidias adheridas

Figura 3. Efecto de colagenasa sobre la capacidad de conidias de M. grisea de adherirse a superficies hidrofóbicas. T1. Conidias + colagenasa incubada por 6 horas T2. Conidias incubadas por 6 horas y luego adición de colagenasa; T3. Conidias incubadas por 6 horas en agua destilada.

\subsection{Efecto de la enzima colagenasa en la patogénesis del hongo Magnaporthe grisea en plántulas de arroz}

Los resultados de este estudio indican que la enzima colagenasa tiene un efecto adverso sobre la patogenicidad del hongo $M$. grisea. Las hojas tratadas solamente con solución de conidias, mostraron síntomas evidentes de la enfermedad presentando manchas grandes de color gris redondas con un halo amarillo. De acuerdo a la escala utilizada en el estudio, este tipo de síntoma corresponde al nivel 4 de la escala. (Figura 4)

En el segundo tratamiento, las hojas que fueron inoculadas con conidias + colagenasa, mostraron pequeñas lesiones, las cuales en algunos casos no eran claramente visibles. Este tipo de síntoma fue ubicado en el nivel 2 de la escala utilizada. Finalmente, el control en el que se utilizó agua destilada no presentó ninguna evidencia de síntomas de la enfermedad, por lo que fue colocado en el nivel 0 de la escala de severidad.

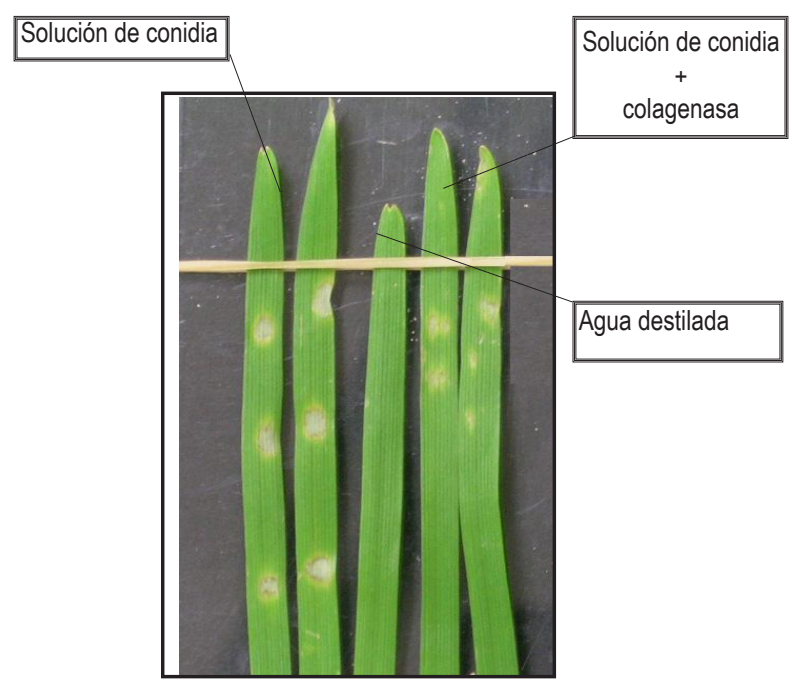

Figura 4. Efecto de la enzima colagenasa sobre la patogenicidad de M. grisea sobre plantulas de arroz 


\section{CONCLUSIONES}

El $78 \%$ de las conidias del hongo $M$. grisea, fueron capaces de producir apresorios cuando fueron colocadas en agua destilada sobre una superficie hidrofóbicas. Sin embargo, las conidias de este hongo no produjeron apresorios cuando fueron colocados sobre superficies hidrofílicas.

La adición de cAMP a una superficie hidrofílica restaura la capacidad de las conidias de producir apresorios, incrementándose de 0 a $69 \%$. Este comportamiento presupone que cAMP induce la formación de apresorios, aun en superficies donde normalmente los hongos no los forman. La germinación, no se vio afectada por las dos superficies utilizadas.

La enzima colagenasa tiene un efecto directo sobre la capacidad de las conidias de $M$. grisea de adherirse a superficies artificiales. Cuando a la solución de conidias le fue aplicado colagenasa después de 6 horas de incubación, hubo una reducción de $61 \%$ en comparación con el tratamiento testigo sin colagenasa. Esta reducción fue más evidente, cuando la solución de conidias fue incubada por 6 horas junto con la colagenasa, ésta última experimentó una reducción de 97\% en comparación con el control sin colagenasa.

Esta enzima, también incide en la severidad de la enfermedad causada por $M$. grisea. En una escala de 0 a 5 donde 0 es no síntoma y 5 es lámina foliar completamente marchita, la colagenasa redujo la severidad de la enfermedad de síntomas con un nivel 4 (Lesiones grandes) en las lesiones hasta lesiones con un nivel 2 (pequeños puntos café).

\section{AGRADECIMIENTO}

El autor agradece a la Oficina en Nicaragua de la Agencia Internacional de Cooperación Japonesa (JICA) por el financiamiento de este estudio. También agradece a la M.Sc. Carmen Marina Rizo y M.Sc Cony Narváez, por sus valiosas sugerencias y correcciones a este documento

\section{BIBLIOGRAFÍA}

1. Bonman, J.M. (1992). Blast. P14-17. in: Compendium of Rice diseases. 2dn edition. St. Paul, MN: American Phytopathological Asociation Press.

2. Bourett, T.M., Picollelli, M.A. and Howard, R.J. (1993). Postembedment labeling of intracellular concanavalin A-binding sites in freeze-substituted fungal cells. Exp. Mycol. 17:223-35
3. Bourett,TM. and Howard, R.J. (1990). In vitro development of penetration structures in the rice blast fungus Magnaporthe grisea. Can. J. Bot. 68:329-42

4. Hamer, J.E, Howard, R.J., Chumley, F.G. and Valent, B. (1988). A mechanism for surface attachment in spores of a plant pathogenic fungus. Science 239:288-90

5. Chaky, J., Anderson, K., Moss, M., and Vaillancourt, L. (2001). Surface hydrophobicity and surface rigidity induce spore germination in Colletotrichum graminicola. Phytopathology 91:558-564.

6. Dean, R. A. (1997). Signal pathways and appressorium morphogenesis. Annu. Rev. Plant Pathol. 35:211-234.

7. FAO. (2002). Concern about rice production practices. Available from

http://www.fao.org/english/newsroom/news/2002/7538en.html.

8. Gilbert, R. D., Johnson, A. M., and Dean, R. A. (1996). Chemical signals responsible for appressorium formation in the rice blast fungus Magnaporthe grisea. Physiol. Mol. Plant Pathol. 48:335-346.

9. IRRI. (2003). Welcome to rice doctor. 4 A.D. Available from http://www.knowledgebank.irri.org/riceDoctor_MX/ Welcome_to_Rice_Doctor.htm

10. Kronstad, J.W. (1997). Virulence and cAMP in smuts, blasts and blights. Trends Plant Sci. 2, 193-199.

11. Lee Y-H and Dean RA. (1994). Hydrophobicity of contact surface induces appressorium formation of Magnaporthe grisea. FEMS Microbiol. Lett. 115:71-76

12. Lee $Y-H$, Dean RA. (1993). cAMP regulates infection structure formation in the plant pathogenic fungus Magnaporthe grisea. Plant Cell 5:693-700

13. Podila, G. K., Rogers, L. M., and Kolattukudy, P. E. (1993). Chemical signals from avocado surface wax trigger germination and appressorium formation in Colletotrichum gloeosporioides. Plant Physiol. 103:267272.

14. Soares, D. M., S. M. Teixeira, and M. F. Castro. (1990). Conjuntura da produção de arroz no Brasil. In: Reunião Nacional de Pesquisa de Arroz. Goiânia, Brasil. 